\title{
Matemática aplicada ao estudo da área ocupada pelo crescimento de micro-organismos como ferramenta para o ensino da função exponencial
}

\author{
Elizangela K. S. Goldoni
}

\section{Resumo}

O estudo de funções no Ensino Médio, praticado sob a perspectiva apenas da matemática teórica, pode-se constituir em obstáculo de difícil transposição no que diz respeito à compreensão de seus conceitos e aplicações. No processo de ensino e aprendizagem dos fundamentos da função exponencial, objeto de estudo deste artigo, tem-se constatado, por meio de avaliações e relatos de alunos, que o tratamento apenas teórico da expressão algébrica associada a essa função pode limitar o desenvolvimento do seu aprendizado. Este artigo, oriundo de uma experiência realizada em sala de aula com alunos da $1^{\mathrm{a}}$ série do Ensino Médio, sugere uma atividade de pesquisa desenvolvida em laboratório, visando compreender e modelar matematicamente o crescimento de micro-organismos coletados da saliva dos alunos e depositados em placas de Petri que foram observadas durante dez dias com a finalidade de medir o crescimento da colônia. Após esse período, foi realizada a análise dos dados aferidos e com o apoio dos programas Excel e GeoGebra, feita a modelagem matemática que se provou fundamentar nos conceitos da função exponencial apresentando suas características, o que proporcionou um aprendizado mais significativo para os alunos envolvidos no experimento. Destaca-se então, o uso do ambiente laboratorial para a construção desse conhecimento, possibilitando ao aluno superar suas limitações e refletir sobre a aplicabilidade dos conceitos aprendidos.

Palavras-chave: Matemática aplicada; Laboratório; Função Exponencial; Modelagem matemática.

\section{Abstract}

The study of functions in High School, practiced from the perspective of only theoretical mathematics, can constitute an obstacle of difficult transposition with respect to the understanding of its concepts and applications. In the process of teaching and learning the fundamentals of the exponential function, object of study of this article, it has been verified, through student evaluations and reports, that the treatment purely theoretical of the algebraic expression associated with this function can limit the development of its learning. This article, from a classroom experience with students of the 1st grade of High School, suggests a research activity developed in laboratory, seeking to understand and mathematically model the growth of microorganisms collected from students' saliva and deposited on petri dishes that were observed during ten days with the purpose of measuring the growth of the colony. After this period, the data analyzed were analyzed and with the support of the Excel and GeoGebra programs, made the mathematical modeling that 
proved to be based on the concepts of the exponential function presenting its characteristics, which provided a more meaningful learning for the students involved in the experiment. We highlight the use of the laboratory environment to build this knowledge, enabling the student to overcome their limitations and reflect on the applicability of the concepts learned.

Keywords: Applied mathematics; Laboratory; Exponential Function; Mathematical modeling.

\section{Introdução}

A abordagem de conteúdos de forma mecanizada e com pouco aprofundamento pode levar o aluno a desenvolver de forma superficial e apenas com ideia de correspondência o domínio de técnicas, fórmulas e procedimentos de manipulações simbólicas e numéricas. Desta forma, inibe-se o desenvolvimento do pensamento complexo, que possibilita a busca e a análise de diferentes tipos de relações, representações e argumentações que podem ser estabelecidas dentro do estudo e aplicação de um conteúdo.

Segundo Maria Salett Biembengut ${ }^{1}$, não se pode negar que boa parte dos professores procura meios eficazes para que seus alunos aprendam. Porém, a estrutura educacional com currículo fragmentado em várias disciplinas, cada uma sob a responsabilidade de um professor que deverá cumprir seu programa em horário e período determinado, dificulta a abordagem mais abrangente e interdisciplinar de cada conteúdo, não contribuindo para que os estudantes percebam a realidade e se interessem pelas questões do meio.

Após pesquisar e analisar várias metodologias utilizadas na área acadêmica, tais como aprendizagem baseada em problemas (PBL), baseada em projetos, aprendizagem entre times (TBL), sala de aula invertida, entre outras, vislumbrou-se as ideias que partem do pressuposto de que na prática aprende-se melhor, propondo metodologias ativas ${ }^{2}$ tais como o "faça você mesmo", o "movimento maker", o "mão na massa". Mas como trazer esse conceito e essas metodologias para as aulas de matemática?

Biembengut, ressalta que a Matemática, enquanto alicerce de quase todas as áreas do conhecimento permite desenvolver os níveis cognitivo e criativo, e defende sua utilização como meio para ajudar o aluno a desenvolver a habilidade de criar, resolver problemas e modelar. Dessa forma, o uso dos ambientes laboratoriais unido à aprendizagem baseada na modelagem matemática, por exemplo, pode permitir essa abordagem mais ativa dentro da sala de aula. Nesses ambientes, pode-se explorar diversos conceitos investigando a relação de dependência entre situações do cotidiano, possibilitando ao aluno aplicar os conteúdos estudados, fazendo as devidas conexões algébricas, o que o levará ao aprofundamento dos conhecimentos adquiridos durante o processo de ensinoaprendizagem.

Partindo desse pressuposto iniciou-se uma pesquisa sobre a possibilidade de aplicar a modelagem matemática no estudo de Funções Exponencias, conteúdo trabalhado na $1^{\mathrm{a}}$ série do Ensino Médio. Foi então constatado que era possível aplicar os fundamentos dessa função e modelar matematicamente diversas situações, tais como: decaimento radioativo e o método do carbono-14, crescimento populacional, magnitude aparente estelar, estudo da pressão atmosférica, estudo do crescimento de

\footnotetext{
${ }^{1}$ BIEMBEnGUT, Maria Salett ; HEIN, Nelson Modelagem matemática no ensino médio. Contexto. 5 ed, São Paulo, 2018.

${ }^{2}$ HOHENWARTER, Markus, HOHENWARTER, Judith. Trad. Antonio Ribeiro.Ajuda GeoGebra - manual Oficial da Versão 3.2. Disponível em: <http://www.geogebra.org>. Acesso em: 05 de maio de 2018.
} 
colônias de micro-organismos, entre outros. No caso desse último, se coletarmos um fungo e observarmos o crescimento da sua colônia, é possível coletar dados e estudar as variações identificando a função que relaciona as grandezas aferidas. Dessa forma têm-se dados suficientes para modelar matematicamente o crescimento da colônia, possibilitando o estudo da expressão algébrica e os gráficos associados a ela, trazendo ao aluno a oportunidade de um estudo aprofundado e aplicado das relações resultantes.

Em vista disso, este artigo apresenta uma atividade, desenvolvida em laboratório, cujo objetivo é auxiliar o professor de Matemática do ensino médio no tratamento do conceito da função exponencial, baseado na perspectiva de mostrar ao aluno como modelar matematicamente situações do seu cotidiano, propondo a construção do conhecimento através de atividades que visam explorar ideias intuitivas de variação e dependência entre os objetos estudados. $\mathrm{O}$ experimento a seguir apresenta condições para que o professor possa trabalhar todos os conceitos relacionados ao estudo da função exponencial.

\section{Conceitos introdutórios}

Entende-se por função, toda relação de dependência entre duas variáveis. A função denominada como exponencial tem como principal característica o aparecimento da variável x no expoente. E assim como toda função, a exponencial também obedece a uma lei de formação.

Observe a notação: $f: \mathbb{R} \rightarrow \mathbb{R}$ tal que $f(x)=a^{x}$, com $0<a \neq 1$.

Sua representação gráfica pode ser definida através de duas situações. Veja os exemplos abaixo.

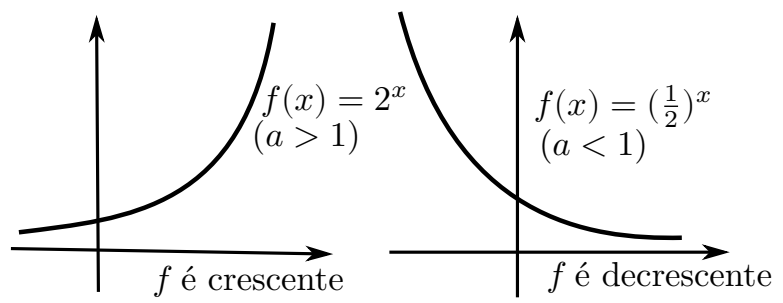

Figura 1: Exemplo de gráfico de funções exponenciais crescentes e decrescentes.(BIZELLI, 2018)

As funções exponenciais possuem diversas aplicações no cotidiano, em especial na Ciência e na indústria. São geralmente utilizadas para representar o crescimento ou decrescimento de dados analisados, expressando situações onde ocorrem grandes variações em curtos períodos, tais como representação de rendimentos financeiros capitalizados por juros compostos, no decaimento radioativo de substâncias químicas, desenvolvimento de bactérias, fungos e micro-organismos, crescimento populacional, entre outras situações, o que faz acreditar que a melhor forma de estudá-la é através de laboratórios.

\subsection{O crescimento de micro-organismos}

Micro-organismos são uma forma de vida que não pode ser visualizada sem auxílio de um microscópio. Tais seres diminutos podem ser encontrados no ar, no solo, e, inclusive, no homem, tais como 
bactérias, fungos, vírus e parasitas. Seu crescimento dá-se por meio de colônias que se tornam visíveis a olho nu. Algumas vezes, os cientistas criam suas próprias colônias para estudar um tipo particular de micro-organismo usando um meio de cultura. Algumas colônias crescem em padrões ondulados, a depender da disponibilidade de nutrientes disponível no seu meio de cultura.

Em geral, pode-se observar quatro fases de crescimento: a primeira fase, de latência (Lag), dáse quando o micro-organismo está se adaptando ao meio de cultura; a segunda fase, é a fase exponencial (Log), na qual o micro-organismo já está adaptado ao ambiente em que está exposto e dirige todo o seu metabolismo para a reprodução; a terceira fase, é a chamada estacionária, na qual os nutrientes já começam a ficar escassos e a taxa de reprodução é equivalente à taxa de mortes; e por fim a quarta e última fase, chamada fase de declínio, na qual a quantidade de micro-organismos que morrem torna-se progressivamente superior àquela dos que surgem.

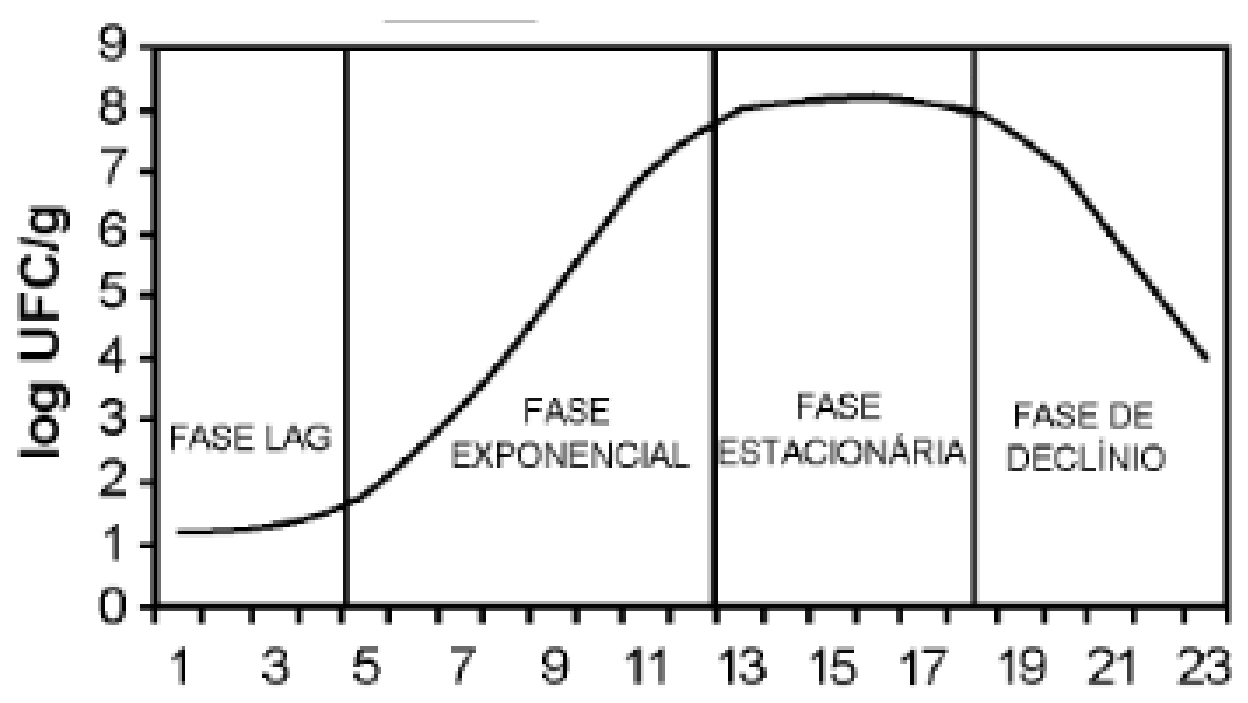

Figura 2: Exemplo de curva de crescimento típica de micro-organismos.(ROBAZZA, 2010)

\section{Procedimento e Análise}

A maioria dos livros didáticos, tais como Matemática: ensino médio de Vanderlei Nemitz, $360^{\circ}$ matemática fundamental de José Ruy Giovanni, entre outros, apresenta esse conteúdo de forma exclusivamente teórica, apenas citando algumas de suas aplicações. Inicialmente revisam as regras de potenciação e logo em seguida apresentam os conceitos e propriedades da função exponencial, concluindo com uma bateria de exercícios que exigem do aluno a associação entre os conceitos apresentados e suas aplicações. Mas como ajudar esse aluno a fazer tal conexão, se tudo fora visto apenas teoricamente até então?

Desse modo e em síntese, sugere-se a intervenção laboratorial, propondo o estudo do crescimento de uma colônia de micro-organismos, visando levar o aluno a refletir sobre a conexão entre os 
conteúdos que serão apresentados ao longo desse laboratório e sua aplicação prática, a fim de modelar matematicamente o experimento realizado. Foi proposta, então, atividade de laboratório investigativa, entendida aqui como aquela que apresenta condições de estudo prático e manipulação do objeto estudado.

O objetivo da atividade foi apresentar uma situação problema, que aparece com muita frequência em atividades teóricas propostas nos livros didáticos, de tal forma que os estudantes obtivessem uma gama razoável de elementos para análise, a fim de observar os resultados e desenvolver estratégias de generalizações, possibilitando ao final da pesquisa o desenvolvimento de modelos matemáticos que representassem o estudo realizado. Para desenvolver o trabalho, os alunos foram divididos em pequenas equipes (no máximo quatro integrantes), dispuseram do laboratório de química e de informática, além de outros recursos como tablets e calculadoras, além do software GeoGebra. Na sequência, será apresentado o passo a passo do experimento, que foi dividido em algumas fases, bem como a análise de um recorte da atividade realizada pelos alunos da $1^{\mathrm{a}}$ série do Ensino Médio do Colégio FAAT, situado na cidade de Atibaia, estado de São Paulo.

$1^{\text {a }}$ fase: A primeira atividade foi diagnosticar quais conceitos básicos, necessários para a análise do experimento deveriam ser retomados com os alunos. Para tanto foi utilizada uma avaliação diagnóstica contendo questões que abordassem as regras da potenciação e conceitos geométricos sobre área e comprimento de circunferência, visto que seriam importantes para coleta e análise dos dados. Após a realização da avaliação, foi divulgado o resultado de cada questão e feita a discussão das mesmas, retomando assim os conceitos necessários. A maior dificuldade apresentada pelos alunos foi sobre as regras para se trabalhar com expoente fracionário e/ou negativo e em especial para análise inversa das propriedades da potência

$2^{\text {a }}$ fase: A intervenção de um professor de Biologia, é interessante nesta fase, visto que além de ser necessário obter algum conhecimento básico sobre microrganismos e meio de cultura, também é uma excelente oportunidade para o trabalho interdisciplinar. Aqui foi então definido quais seriam os procedimentos para a realização da coleta dos micro-organismos e preparação do meio de cultura. A partir do estudo realizado juntamente com o professor de Biologia, considerou-se que o meio de cultura semissólido seria o mais propício para a realização do experimento, e a coleta deveria ser feita através da saliva.

$3^{\text {a }}$ fase: Nesta fase iniciou-se o procedimento laboratorial. As etapas destacadas a seguir foram realizadas por cada equipe, composta por no máximo quatro alunos.

\subsection{Etapa 1 - Preparando o meio de cultura}

Cada equipe recebeu um cotonete que deveria ser esfregado na bochecha de um dos membros até ficar bem úmido e depois passado levemente na superfície da gelatina, infectando assim o meio de cultura preparado. 


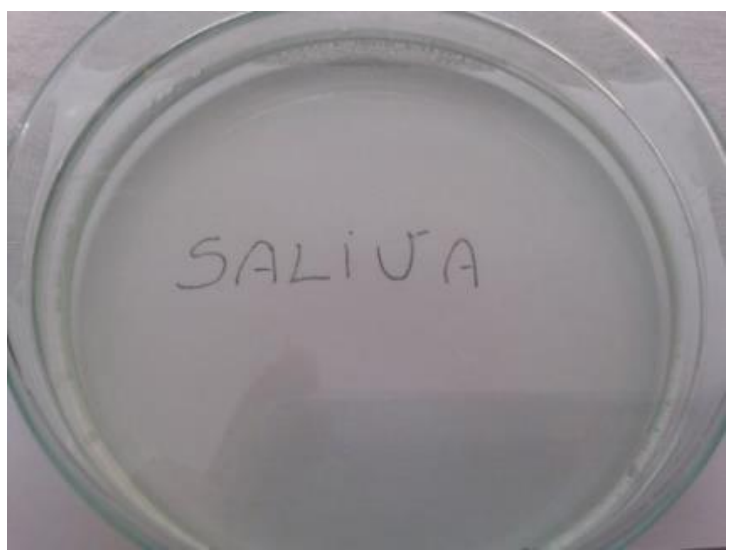

Figura 3: infectando o meio de cultura.(Própria autoria)

Para preparar o meio de cultura foi utilizado um pacotinho de gelatina sem sabor e incolor, meio litro de água fervente, duas colheres de açúcar e duas placas de Petri , devidamente esterilizadas. A gelatina e o açúcar foram dissolvidos em água fervida, obtendo-se um líquido homogêneo. A mistura foi despejada em uma das placas e leveda à geladeira até endurecer. A placa preparada irá receber os micro-organismos que serão coletados da saliva dos alunos através de um cotonete.

\subsection{Etapa 2 - Colhendo os micro-organismos}

Cada equipe recebeu um cotonete que deveria ser esfregado na bochecha de um dos membros até ficar bem úmido e depois passado levemente na superfície da gelatina, infectando assim o meio de cultura preparado.

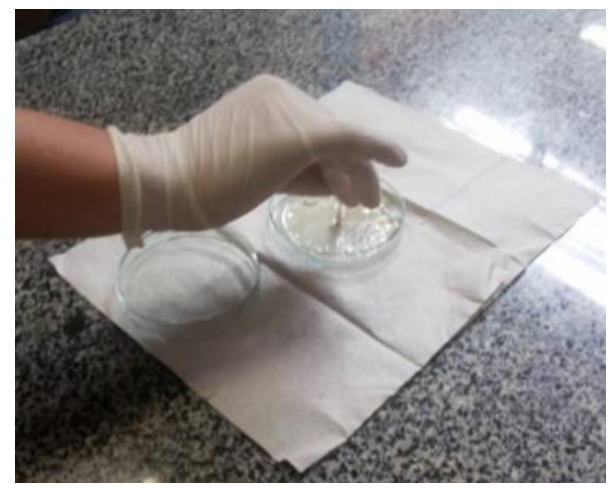

Figura 4: Infectando o meio de cultura.(Própria autoria) 
Após a coleta dos micro-organismos lacrou-se a placa utilizando fita adesiva transparente e a deixou em local com pouca luz.

Cada equipe ficou responsável por coletar os dados seguindo as orientações do relatório disponibilizado no Anexo 1, deste artigo. As placas ficaram guardadas no laboratório de química do Colégio e no fim de semana um aluno da equipe ficou responsável por levá-las para casa e dar continuidade à coleta de dados.

\subsection{Etapa 3 - Aferindo as medidas}

Ao longo de 10 dias cada equipe deveria medir o diâmetro da circunferência da colônia, visto que esta crescia circularmente. Abaixo pode-se observar as fotos e dados coletados por uma das equipes. Faz-se necessário ressaltar que várias colônias cresceram no meio preparado e que foi escolhida apenas uma para estudo.

Dia da contaminação

$3^{\circ}$ dia pós-contaminação $1^{\circ}$ dia pós-contaminação

$4^{\circ}$ dia pós-contaminação $2^{\circ}$ dia pós-contaminação

$5^{\circ}$ dia pós-contaminação 
Figura 5: crescimento diário de uma colônia de micro-organismos, registrado por um dos grupos envolvidos no experimento.(Própria autoria)

Após observação do crescimento da colônia e coleta dos dados solicitados no experimento, em sala de aula, as equipes realizaram os cálculos necessários para preencher a tabela disponibilizada no relatório e discutiram os resultados. Cada equipe modelou, manualmente, a função que confirmava o crescimento exponencial da colônia observada, iniciando a próxima etapa do experimento.

\subsection{Etapa 4 - Modelagem matemática do experimento}

Ao observar o crescimento da colônia ao longo de 10 dias foi possível, através da aferição do seu diâmetro, calcular a área de contaminação. Foi considerada aproximação de duas casas decimais. 


\begin{tabular}{|c|c|c|c|c|c|}
\hline & $+C$ & $f_{x}$ & & & \\
\hline$\Delta$ & A & B & C & D & E \\
\hline $\begin{array}{l}1 \\
2 \\
\end{array}$ & $\begin{array}{l}\text { Dia }\left(\mathrm{n}^{\circ}\right)-\text { pós } \\
\text { contaminação }\end{array}$ & $\begin{array}{c}\text { Contaminação } \\
\text { (diâmetro - mm) }\end{array}$ & $\begin{array}{c}\text { Contaminação } \\
\text { (diâmetro - cm) }\end{array}$ & $\begin{array}{c}\text { Contaminacão } \\
\text { (raio/cm) }\end{array}$ & $\begin{array}{l}\text { Área de contaminação } \\
\qquad\left(\mathrm{cm}^{2}\right)\left(\mathrm{A}_{\mathrm{c}}=\pi \mathrm{r}^{2}\right)\end{array}$ \\
\hline 3 & Dia da contaminação & \multicolumn{4}{|c|}{ Fase 1 de crescimento (adaptação ao meio de cultura) } \\
\hline 4 & $1^{\circ} \mathrm{dia}-\mathrm{T}_{2}$ & 13 & 1,30 & 0,65 & 1,33 \\
\hline 5 & $2^{\circ} \mathrm{dia}-\mathrm{T}_{3}$ & 15 & 1,50 & 0,75 & 1,77 \\
\hline 6 & $3^{\circ} \mathrm{dia}-\mathrm{T}_{4}$ & 17 & 1,70 & 0,85 & 2,27 \\
\hline 7 & $4^{\circ} \mathrm{dia}-\mathrm{T}_{5}$ & 20 & 2,00 & 1,00 & 3,14 \\
\hline 8 & $5^{\circ} \mathrm{dia}-\mathrm{T}_{6}$ & 23 & 2,30 & 1,15 & 4,15 \\
\hline 9 & $6^{\circ} \mathrm{dia}-\mathrm{T}_{7}$ & 26 & 2,60 & 1,30 & 5,31 \\
\hline 10 & $7^{\circ} \mathrm{dia}-\mathrm{T}_{8}$ & 29 & 2,90 & 1,45 & 6,6 \\
\hline 11 & $8^{\circ} \mathrm{dia}-\mathrm{T}_{9}$ & 33 & 3,30 & 1,65 & 8,55 \\
\hline 12 & $9^{\circ} \mathrm{dia}-\mathrm{T}_{10}$ & 35 & 3,50 & 1,75 & 9,62 \\
\hline 13 & $10^{\circ} \mathrm{dia}-\mathrm{T}_{11}$ & 37 & 3,70 & 1,85 & 10,75 \\
\hline$\frac{14}{14}$ & $11^{\circ}$ dia $-T_{12}$ & \multicolumn{4}{|c|}{ Iniciou-se a fase estacionária } \\
\hline
\end{tabular}

Figura 6: Dados coletados referentes ao crescimento de uma das colônias estudadas.(Própria autoria)

Com os valores referentes em mãos foi feita a representação gráfica da área contaminada em função do tempo, dado em dias. Os pontos (pares ordenados) foram marcados no plano cartesiano determinando como domínio (x) os dias e como imagem (y) a área de contaminação. Ao unir esses pontos observou-se que o gráfico era semelhante ao gráfico de uma função exponencial. 


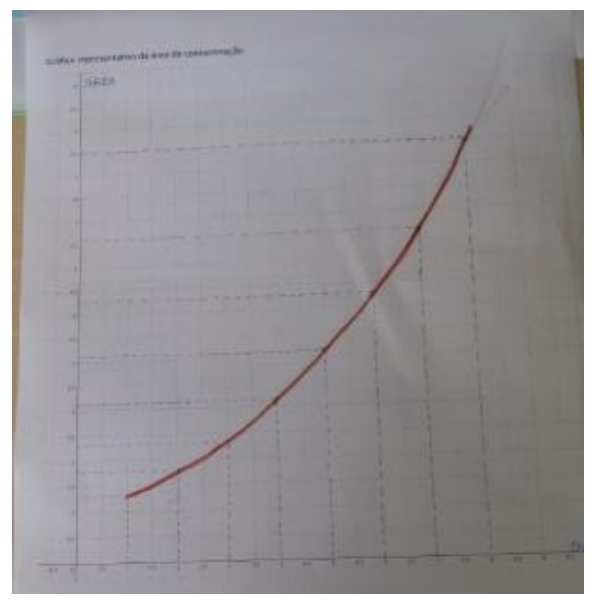

Figura 7: Gráfico construído manualmente por uma das equipes de pesquisa.(Própria autoria)

Após construção do gráfico, os alunos substituíram os valores encontrados, na lei geral da função exponencial, $f(x)=a^{x}$, a fim de determinar o algoritmo que define a curva de crescimento dos micro-organismos estudados. Abaixo observe a modelagem realizada por uma das equipes.

Foram realizadas um total de 10 medições, sendo considerado:

- Fase de adaptação ao meio: dia da contaminação tempo inicial - T1;

- Início da fase exponencial: primeiro dia após contaminação - T2;

- Final da fase exponencial: décimo primeiro dia após contaminação - T11;

- Fase estacionária: após o $11^{\mathrm{O}}$ dia observou-se que a colônia não crescia mais. 


\begin{tabular}{|c|c|c|c|}
\hline & \multicolumn{3}{|c|}{ Cálculo para determinar a função } \\
\hline & \multicolumn{3}{|c|}{$f(x)=a^{\wedge} x$} \\
\hline & $\mathbf{x}$ & $f(x)$ & a \\
\hline Dia da contaminação & **** & *:*** & ***** \\
\hline Análise 20 dia & 2 & 1,33 & 1,1532563 \\
\hline Análise $3 \circ$ dia & 3 & 1,77 & 1,2096445 \\
\hline Análise 40 dia & 4 & 2,27 & 1,2274575 \\
\hline Análise 50 dia & 5 & 3,14 & 1,2571466 \\
\hline Análise 60 dia & 6 & 4,15 & 1,2676753 \\
\hline Análise $7^{0}$ dia & 7 & 5,31 & 1,2693604 \\
\hline Análise $8 \cong$ dia & 8 & 6,74 & 1,2693532 \\
\hline Análise 99 dia & 9 & 8,55 & 1,2692635 \\
\hline Análise $10 \%$ dia & 10 & 10,37 & 1,2635076 \\
\hline \multirow[t]{2}{*}{ Análise 11 ㅇa } & 11 & 11,94 & 1,2528800 \\
\hline & & Moda & 1,27 \\
\hline
\end{tabular}

Figura 8: Dados analisados no Excel para determinação do coeficiente "a".(Própria autoria)

Considerando então o valor da moda encontrada nos dados calculados para o coeficiente "a" obtevese a função exponencial que determinava o crescimento da colônia de micro-organismos em estudo. No exemplo utilizado acima, oriundo do experimento de uma das equipes, observou-se um crescimento exponencial determinado pela função $f(x)=1,27^{x}$. Cada equipe encontrou uma função exponencial diferente, o que determina que a área ocupada por colônias de diferentes microorganismos dá-se em ritmo diferente para cada uma delas.

Com a lei calculada, chegou o momento de validar os resultados obtidos. Os alunos foram motivados a fazer a análise inicialmente no Excel, a fim de determinar a equação do gráfico obtido.

A equipe em destaque neste artigo ao construir o gráfico de dispersão no Excel, obteve a equação dos dados fornecidos, $y=e^{0.239 x}$, o que confirmou a função encontrada nos cálculos manuais, $f(x)=$ $1,27^{x}$, visto que o coeficiente "e" na equação encontrada através do software corresponde ao número de Euler, que vale aproximadamente 2,7. Elevando esse número a $0,239 x$, onde $x$ é determinado pelo dia da contaminação, chega-se a resultados que determinam os valores correspondentes às áreas encontradas para cada dia. 


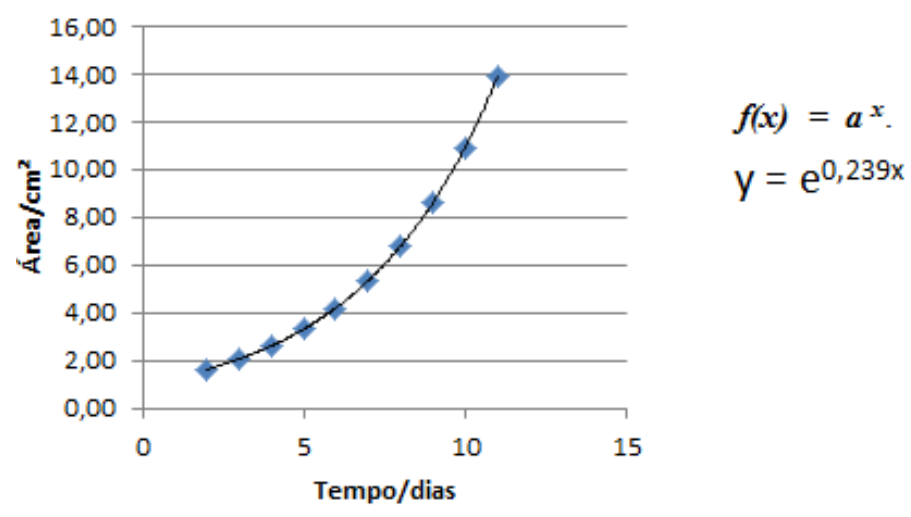

Figura 9: Gráfico do crescimento exponencial da colônia construído no Excel.(Própria autoria)

Após essa análise cada equipe, com o auxílio de tablets, pôde modelar matematicamente, utilizando o programa GeoGebra, a função exponencial encontrada, a fim de observar o comportamento do gráfico da mesma. Aqui vale destacar que tal software associa ferramentas algébricas e geométricas, o que possibilita, em um mesmo ambiente, construir figuras e expressões dinâmicas que representam relações comuns, permitindo sua investigação. Assim, o GeoGebra possibilita representar as diferentes facetas do objeto estudado e suas interações, oferecendo, portanto, possibilidades para a exploração do conteúdo apresentado pelo professor em sala de aula.

Foram, então, inseridos nesse programa os dados coletados e estudados inicialmente, obtendose assim o gráfico que, ao ser comparado com o gráfico construído manualmente, provou-se a exatidão da modelagem, comprovando assim o crescimento exponencial da área ocupada pela colônia observada. 


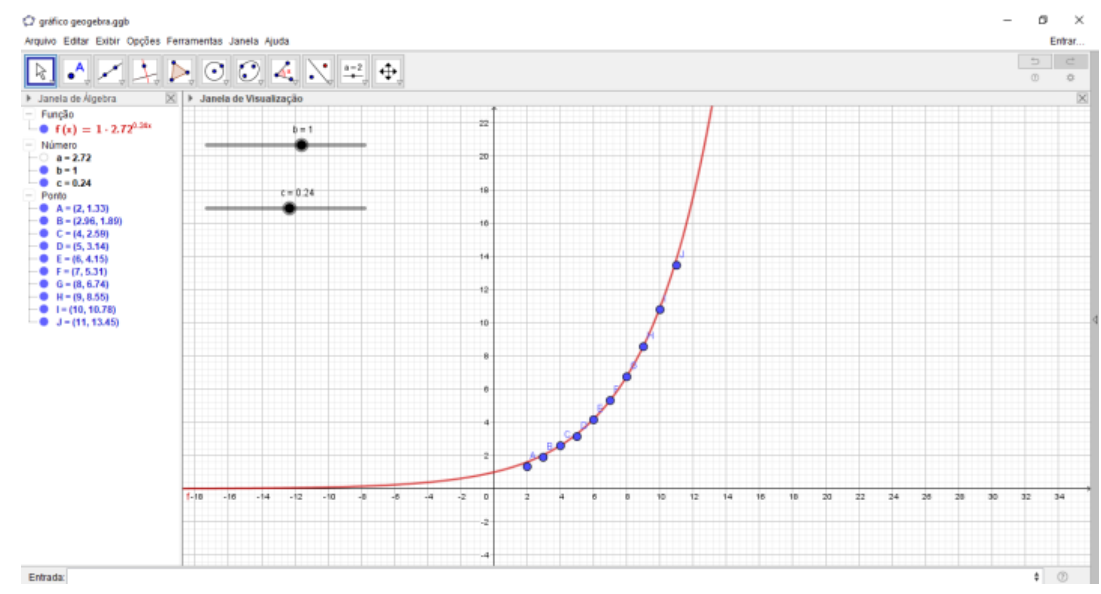

Figura 10: Gráfico do crescimento exponencial da colônia construído no GeoGebra.(Própria autoria)

Logo os resultados obtidos pela modelagem matemática determinada no experimento indicam que o modelo empregado consegue reproduzir a área ocupada pelo crescimento do micro-organismo estudado.

Durante todas as etapas para realização do experimento e da modelagem matemática associada a ele, destaca-se ressaltar que as discussões em torno da construção dos conceitos e fundamentos sobre função exponencial com a turma deram-se de forma construtiva e possibilitaram que o aluno fosse protagonista e capaz de desenvolver um discurso com embasamento muito mais consistente.

\section{Considerações Finais}

O artigo aqui apresentado propõe uma metodologia diferenciada para se trabalhar a Matemática em sala de aula. O uso de laboratórios resulta em aulas dinâmicas e mais apreciáveis, podendo então se tornar um método mais eficaz quando se trata de um aprendizado significativo. O experimento proposto aqui traz a possibilidade de se trabalhar a função exponencial de forma mais envolvente, proporcionando um aprendizado mais significativo para os alunos envolvidos, fato esse observado através da mudança de postura dos mesmos e dos resultados nas avaliações teóricas. Destacase, então, o uso do ambiente laboratorial para a construção desse conhecimento, possibilitando ao aluno superar suas limitações e refletir sobre a aplicabilidade dos conceitos aprendidos, tornando-os mais significativos.

\section{Referências}

[1] BIEMBEnGUT, Maria Salett; HEIN, Nelson. Modelagem matemática no ensino médio. Contexto. 5 ed, São Paulo, 2018. 
[2] BIZELLI, Maria Helena S. S. Conteúdos didáticos digitais para o ensino e aprendizagem de Cálculo. Disponível em: <http://www.calculo.iq.unesp.br/Calculo1/funcao-graficos-exponencial. html>. Acesso em: 10 de janeiro de 2018.

[3] GARAFALO, Débora. "Como as metodologias ativas favorecem o aprendizado". Revista Nova escola, 25 jun 2018. Disponível em: <https://novaescola.org.br/conteudo/11897/ como-as-metodologias-ativas-favorecem-o-aprendizado >. Acesso em: 30 jun 2018.

[4] HOHENWARTER, Markus; HOHENWARTER, Judith. Trad. Antonio Ribeiro. Ajuda GeoGebra - manual Oficial da Versão 3.2. Disponível em: <http://www.geogebra.org>. Acesso em: 05 de maio de 2018.

[5] ROBAZZA, W.S.; TELEKEN, J.T.; GOMES, G.A. Modelagem Matemática do Crescimento de Microrganismos em Alimentos. Sociedade Brasileira de Matemática Aplicada e Computacional. Vol. 11, noํ. 1, p.101-110. 2010. Disponível em: <https://tema.sbmac.org.br/tema/article/ viewFile/117/61>. Acesso em: 13 de maio de 2018.

Elizangela K. S. Goldoni

Colégio FAAT de Atibaia <elizangela.goldoni@hotmail.com>

Recebido: 25/09/2018

Publicado: 01/07/2019 